\title{
Characterization of multidrug-resistant osteosarcoma sublines and the molecular mechanisms of resistance
}

\author{
JIAN-ZENG YANG ${ }^{1}$, SHU-RONG MA ${ }^{2}$, XIAO-LI RONG ${ }^{1}$, MEI-JU ZHU ${ }^{3}$, \\ QIU-YE JI ${ }^{1}$, LING-JIE MENG ${ }^{1}$, YI-YAO GAO ${ }^{1}$, YU-DAN YANG ${ }^{1}$ and YAN WANG ${ }^{1}$ \\ ${ }^{1}$ Scientific Research Center; ${ }^{2}$ Endoscopy Center, China-Japan Union Hospital of Jilin University, Changchun, Jilin 130033; \\ ${ }^{3}$ Department of Gastroenterology and Hypothyroid Surgery, The Fifth Affiliated Hospital of Zhengzhou University, \\ Zhengzhou, Henan 450000, P.R. China
}

Received July 24, 2015; Accepted July 8, 2016

DOI: $10.3892 / \mathrm{mmr} .2016 .5590$

\begin{abstract}
Multidrug resistance (MDR) is a challenge for the treatment of cancer and the underlying molecular mechanisms remain elusive. The current study exposed MG63 osteosarcoma cells to increasing concentrations of vincristine (VCR) to establish four VCR-resistant MG63/VCR cell sublines (MG63/VCR1, 2, 3 and 4). The drug resistance indices (RI) of these sublines was detected with the CCK-8 assay and determined to be163, 476, 1,247, and 2,707-fold higher than that of parental cells, respectively. These sublines also exhibited cross-resistance to doxorubicin, paclitaxel and pirarubicin. With increased RI, the proliferative capacity of these sublines was gradually reduced and cell morphology was also altered, characterized by increased formation of pseudopodia and long cytoplasmic processes at opposite poles. However, the migration capacity and expression of certain drug resistance-associated genes were not in accordance with the increased RI; multidrug resistance protein 1 (MDR1) expression was significantly increased in these sublines compared with parental cells. However, in the highly resistant MG63/VCR3 and MG63/VCR4 cells, MDR-associated protein 1, topoisomerase II and LIM domain kinase 1 levels were significantly reduced compared with the moderately resistant MG63/VCR2 cells. Expression of glutathione $\mathrm{S}$-transferase- $\pi$ mRNA was determined using reverse transcription-quantitative polymerase chain reaction and determined that it was not changed between MG63 and MG63/VCR cells. The data of the present study demonstrated that the molecular alterations of drug resistance may change with the degree of drug resistance. Taking cell morphology into consideration, the intratumor clonal and phenotypic heterogeneity may be responsible for drug resistance. These
\end{abstract}

Correspondence to: Professor Yan Wang, Scientific Research Center, China-Japan Union Hospital of Jilin University, 126 Xiantai Street, Changchun, Jilin 130033, P.R. China

E-mail: wangy01@jlu.edu.cn

Key words: osteosarcoma, chemotherapy, multidrug resistance, vincristine, tumor heterogeneity
MG63/VCR sublines may be a valuable tool to assess drug resistance and the underlying mechanisms, and to identify novel drug resistance-associated genes or strategies to overcome MDR in human osteosarcoma.

\section{Introduction}

Osteosarcoma is a highly malignant bone tumor with widespread histological heterogeneity. Clinically, osteosarcoma lacks biomarkers for early detection and differential diagnosis and has high local aggressiveness with rapid metastatic potential (1). Osteosarcoma is the most common primary bone malignancy and the third most common cancer in adolescence and young adults (2). To date, the cause and risk factors of osteosarcoma remain unclear (2). Depending on the stage at diagnosis, chemotherapy is routinely used to treat patients with advanced osteosarcoma and chemotherapeutic drugs, including methotrexate (MTX), doxorubicin (DOX), vincristine (VCR) and cisplatin are commonly used in the clinic (3-5). Despite advances in preoperative neoadjuvant chemotherapy, osteosarcoma prognosis remains poor due to the development of multidrug resistance (MDR) $(4,6,7)$. Tumor cells may become cross-resistant to a broad spectrum of chemotherapeutic agents following single drug-induced resistance (8). Currently, VCR remains an effective chemotherapeutic agent to control osteosarcoma (5); however, resistance to VCR chemotherapy frequently occurs, as tumor cells often acquire resistance to drugs and develop MDR, resulting in treatment failure. However, the mechanism of drug resistance in osteosarcoma remains elusive. Thus, the current study generated VCR-resistant osteosarcoma sublines and subsequently assessed their characteristics, MDR and the underlying molecular events. Indeed, drug-resistant cell sublines generated by various research groups have reported different and even contradictory results with regards to cell proliferation or migration ability, and cell cycle distribution (9-12). Thus, it is speculated that these contradictions may be caused by the different resistance index and tumor heterogeneity, as the tumor cell phenotypes have different cell subpopulations $(13,14)$. Different mechanisms are responsible for the development of drug resistances at the cellular level, which has long been reported in morphological, transcriptional, genetic 
and epigenetic studies of cancer (15), and recently in genomic studies $(16,17)$. The current study may provide a useful tool for the future study of drug-resistant mechanisms and strategies for identification of novel targets for the treatment of patients with osteosarcoma.

\section{Materials and methods}

Cell lines and culture. The MG63 human osteosarcoma cell line was obtained from the Institute of Biochemistry and Cell Biology, Chinese Academy of Sciences (Shanghai, China) and cultured in high glucose Dulbecco's modified Eagle's medium (H-DMEM; Invitrogen; Thermo Fisher Scientific, Inc., Waltham, MA, USA) supplemented with $10 \%$ fetal bovine serum (FBS; Hyclone, Logan, UT, USA) at $37^{\circ} \mathrm{C}$ in a humidified incubator with $5 \% \mathrm{CO}_{2}$.

Establishment of VCR-resistant cell sublines. To generate VCR-resistant MG63 sublines, we exposed MG63 cells to increasing concentrations of VCR according to a previous study (18). Briefly, parental MG63 cells were initially cultured in H-DMEM containing VCR (New Hualian Pharmaceutical Co., Shanghai, China) at a concentration of $10 \mathrm{ng} / \mathrm{ml}$ for $72 \mathrm{~h}$. Surviving cells were collected and cultured in VCR-free medium for 1-2 weeks and then further cultured with VCR-containing H-DMEM. This procedure was repeated for five or more times until the majority of cells survived in the drug-containing medium. Subsequently, cells were exposed to increasing concentrations of VCR $(10,20,30,50,75,100$, $200,300,500,800,1,000$ and $2,500 \mathrm{ng} / \mathrm{ml})$. At the high VCR concentrations, 4-6 weeks were required to establish adequate growth. Clones selected at 100, 500, 1,000 and 2,500 ng/ml VCR were re-purified for the subsequent experiments and termed MG63/VCR1, MG63/VCR2, MG63/VCR3 and MG63/VCR4 respectively, collectively termed MG63/VCR cells. Prior to each experiment, MG63 and MG63/VCR cells were maintained in a drug-free medium and subcultured at least three times.

Colony formation assay. Cells in an exponential growth phase were seeded in triplicate in a 6-well plate at a density of 500 cells per well in H-DMEM containing 10\% FBS for 2 weeks. Colonies were counted following fixation with methanol and stained with $0.1 \%$ crystal violet (Sigma-Aldrich, St Louis, MO, USA) in phosphate-buffered saline (PBS) for $15 \mathrm{~min}$. The experiments were repeated at least once.

Cell viability assay. The Cell Counting Kit-8 (CCK-8; Dojindo Molecular Technologies, Inc., Kumamoto, Japan) was applied to assess the effect of VCR, MTX, pirarubicin (THP), DOX (all from New Hualian Pharmaceutical Co.), paclitaxel (PTX), and gemcitabine (GEM) (both from Laboratories Pierre Fabre, Paris, France). Specifically, mono-dispersed cells in the exponential growth phase were plated into 96-well plates at a density of $8 \times 10^{3}$ cells per well and cultured in $100 \mu 1 \mathrm{H}$-DMEM supplemented with $10 \%$ FBS for $24 \mathrm{~h}$. Subsequently, the culture medium was replaced with medium containing serial dilutions of various drug formulations as follows: $125,25,5,1,0.2,0.04$, $0.008,0.0016 \mu \mathrm{g} / \mathrm{ml}$ and drug-free medium used as a control. After $48 \mathrm{~h}$, the medium was replaced with $10 \%$ CCK- 8 and cells were incubated at $37^{\circ} \mathrm{C}$ for $2 \mathrm{~h}$. Subsequently, the plates were measured at the wavelength of $450 \mathrm{~nm}$ using a plate reader (UV8100D; LabTech, Inc., Hopkinton, MA, USA). The inhibition ratio was calculated using the following formula: Inhibition ratio $=\left(\mathrm{OD}_{\text {control }}-\mathrm{OD}_{\text {experiment }}\right) / \mathrm{OD}_{\text {control }} \times 100 \%$. Based on this the 50\% inhibitory concentration $\left(\mathrm{IC}_{50}\right.$ ) was calculated by the GraphPad Prism 5 (GraphPad Software, USA) and the resistance indices (RI) was calculated as follows: $\mathrm{RI}=\mathrm{IC}_{50 \mathrm{MG} 63 / \mathrm{VCR}} / \mathrm{IC}_{50 \mathrm{MG} 63}$.

Flow cytometry cell cycle assay. MG63 and MG63/VCR cells following different treatments were collected following trypsin digestion, washed with PBS and fixed in $70 \%$ ethanol at $4^{\circ} \mathrm{C}$ overnight. The fixed cells were then washed again with cold PBS and stained with $50 \mu \mathrm{g} / \mathrm{ml}$ DNA-binding dye propidium iodide (PI; Sigma-Aldrich) and $1.0 \mu \mathrm{g} / \mathrm{ml}$ RNase A (Invitrogen; Thermo Fisher Scientific, Inc.) for $30 \mathrm{~min}$ at $37^{\circ} \mathrm{C}$ in the dark. Cells were then analyzed for cell cycle distribution using a flow cytometer (BD LSRII; BD Biosciences, San Jose, CA, USA) with excitation at $485 \mathrm{~nm}$ and emission at $620 \mathrm{~nm}$. Each experiment was performed in triplicate and repeated three times. FACSDiva 6.0 (BD Biosciences) was used to analyze the cell cycle distribution.

Cell migration assay. MG63 and MG63/VCR1-4 cells were seeded in 24-well culture plates at a density of $8 \times 10^{4}$ cells per well When the cell reached $\sim 90 \%$ confluency a $20 \mu$ l pipette tip was used to make a scratch through the cell monolayer and cells were washed three times with PBS and subsequently cultured in H-DMEM without serum for up to $24 \mathrm{~h}$. At the end of each experiment, images were captured using a fluorescence IX70 microscope (Olympus Corporation, Tokyo, Japan) and analyzed. The migration abilities were quantified by measuring the area of the scratched regions using ImageJ software (imagej.nih.gov).

$R N A$ isolation and reverse transcription-quantitative polymerase chain reaction (RT-PCR). Total cellular RNA was isolated using Trizol reagent (Invitrogen; Thermo Fisher Scientific, Inc.) and RT was performed to obtain cDNA using the EX Tag kit (Takara Biotechnology Co., Ltd., Dalian, China) according to the manufacturer's protocols. Subsequently, PCR was performed using SYBR ${ }^{\circledR}$ Premix Ex Taq ${ }^{\mathrm{TM}}$ II (Takara) with an ABI7500 Fast Real-Time PCR System (Applied Biosystems; Thermo Fisher Scientific, Inc.). Relative expression of each gene was calculated using the $2^{-\Delta \Delta C q}$ method (19). The conditions used for qPCR were as follows: Denaturation at $98^{\circ} \mathrm{C}$ for $10 \mathrm{sec}$; annealing at $54-62^{\circ} \mathrm{C}$ for $30 \mathrm{sec}$; and elongation at $72^{\circ} \mathrm{C}$ for $1 \mathrm{~min}$. PCR products were visualized by electrophoresis on $1.5 \%$ agarose gel stained with GelRed ${ }^{\mathrm{TM}}$ (Biotium, Inc., Hayward, CA, USA). Images were obtained using the Bio-Rad Gel Doc XR+ (Bio-Rad Laboratories, Inc., Hercules, CA, USA) and analyzed using the Image $\mathrm{Lab}^{\mathrm{TM}}$ software (version 4.0; Bio-Rad Laboratories, Inc.). PCR primers used are as follows: Multidrug resistance protein 1 (MDR1), forward 5'-ATATCAGCAGCCCACATCAT-3', reverse 5'-GAA GCACTGGGATGTCCGGT-3'; MDR-associated protein 1 (MRP1), forward 5'-GTACATTAACATGATCTGGTC-3', reverse 5'-CGTTCATCAGCTTGATCCGAT-3'; glutathione S-transferase- $\pi$ (GST- $\pi$ ), forward 5'-ATGCTGCTGGCAGAT 


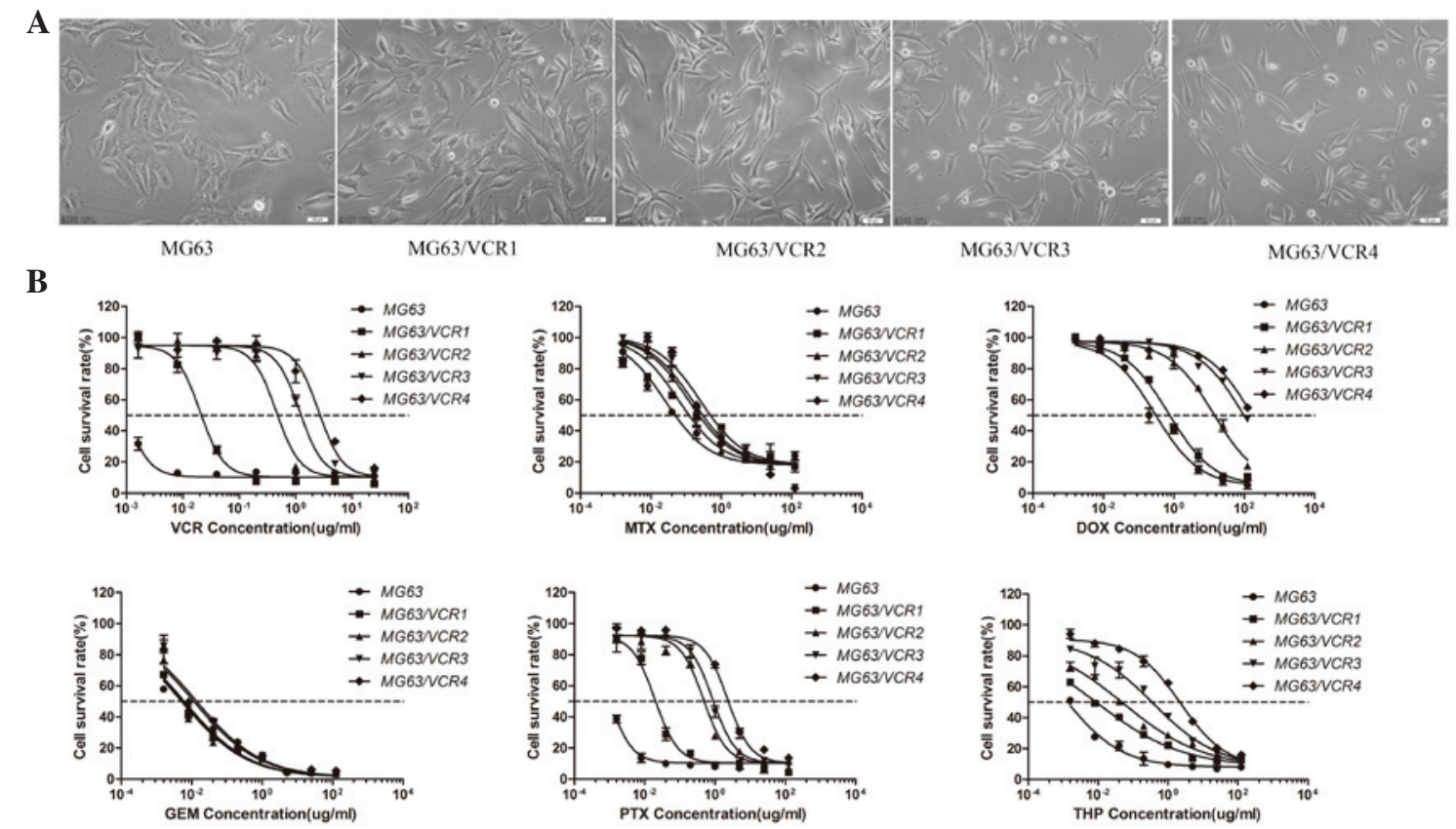

C

D

\begin{tabular}{lccccc}
\hline \multirow{2}{*}{ Drugs } & \multicolumn{5}{c}{ IC50 (mean \pm SD, ng/ml) } \\
\cline { 2 - 6 } & MG63 & MG63/VCR1 & MG63/VCR2 & MG63/vCR3 & MG63/VCR4 \\
\hline VCR & $0.9521 \pm 0.104$ & $155.3 \pm 16.9$ & $453.4 \pm 49.4$ & $1182 \pm 128$ & $2578 \pm 283$ \\
MrX & $25.39 \pm 3.29$ & $63.02 \pm 9.04$ & $120.4 \pm 16.1$ & $153.5 \pm 19.9$ & $254.8 \pm 35.4$ \\
DOX & $289.8 \pm 33.4$ & $720.1 \pm 83.2$ & $13460 \pm 1540$ & $92330 \pm 42700$ & $160800 \pm 20400$ \\
GEM & $4.883 \pm 0.886$ & $5.371 \pm 0.976$ & $5.838 \pm 1.06$ & $10.91 \pm 2.02$ & $13.14 \pm 2.42$ \\
PTX & $1.24 \pm 0.140$ & $19.62 \pm 2.17$ & $470.3 \pm 53.2$ & $862.5 \pm 95.2$ & $2292 \pm 256$ \\
THP & $1.691 \pm 0.209$ & $7.81 \pm 0.922$ & $40.06 \pm 4.59$ & $588.5 \pm 66.4$ & $1962 \pm 215$ \\
\hline
\end{tabular}

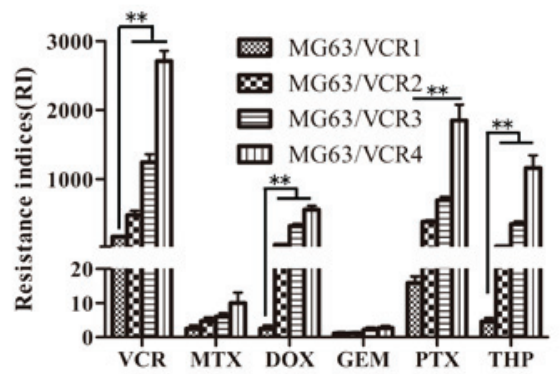

Figure 1. Establishment of multidrug resistant osteosarcoma sublines. (A) Morphology of MG63 and MG63/VCR cells in the exponential growth phase under an inverted microscope with an original magnification of $\mathrm{x} 200$. Scale bar $=50 \mu \mathrm{m}$. (B) The concentration at which each drug produced $50 \%$ inhibition of growth $\left(\mathrm{IC}_{50}\right)$ was estimated by the relative survival curve. Graphs were constructed using GraphPad with the equation of global nonlinear regression. X values are the logarithms of molar concentration. Y values are responses to drug treatment. (C) $\mathrm{IC}_{50}$ values of sensitive and resistant cell sublines. (D) Drug resistance indices were determined by the ratio of IC50 values of MG63/VCR cells to that of MG63 cells. Data represent the mean \pm SD of 5 independent experiments. ${ }^{* *} \mathrm{P}<0.01$ vs. the MG63/VCR1 group. VCR, vincristine; MTX, methotrexate; DOX, doxorubicin; GEM, gemcitabine; PTX, paclitaxel; THP, pirarubicin; SD, standard deviation.

CAG-3', reverse 5'-GTAGATGAGGGAGATGTATTTGCA-3'; and $\beta$-actin, forward 5'-CTGGGACGACATGGAGAAAA-3', reverse 5'-AAGGAAGGCTGGAAGAGTGC-3'.

Intracellular DOX accumulation. Cells in an exponential growth phase were plated into 60-mm Petri dishes and treated with or without $1.5 \mu \mathrm{M}$ DOX with or without $5 \mu \mathrm{M}$ verapamil for $3 \mathrm{~h}$ at $37^{\circ} \mathrm{C}$. Subsequently, cells were washed three times with ice-cold PBS and imaged under an inverted fluorescence microscope (IX70; Olympus Corporation) with a suitable filter at x200 magnification. Additionally, the cells were analyzed using flow cytometry to measure DOX auto-fluorescence. Cells were centrifuged and suspended in ice-cold PBS and the mono-dispersed cells were analyzed by a flow cytometer (BD Biosciences) according to the method a previous study (20). Cell fluorescence was measured in duplicate at each time point, and all experiments were repeated three times.

Statistical analysis. All data are expressed as the mean \pm standard deviation. Statistically significant difference was assessed using the unpaired $t$-test to compare means between two groups, or one-way analysis of variance to compare the mean values among three or more groups using GraphPad Prism software (version 5.04 for Windows; GraphPad Software, Inc., La Jolla, CA, USA). P $<0.05$ was considered to indicate a statistically significant difference.

\section{Results}

Characterization of VCR-resistant osteosarcoma cell sublines. The VCR-resistant sublines exhibited specific morphological characteristics when observed using inverted microscope. For example, although MG63 and MG63/VCR cell lines can proliferate and attach to the bottom of cell culture dishes, MG63/VCR cells exhibited irregular shapes with an increased triangular appearance and size, with some giant and small cells. MG63/VCR cells were often spindle shaped with increased formation of pseudopodia compared with the MG63 cells. The ratio of the long and short axis gradually increased with increased drug RI (Fig. 1A). By contrast, parental MG63 cells were relatively uniform in size, 
A

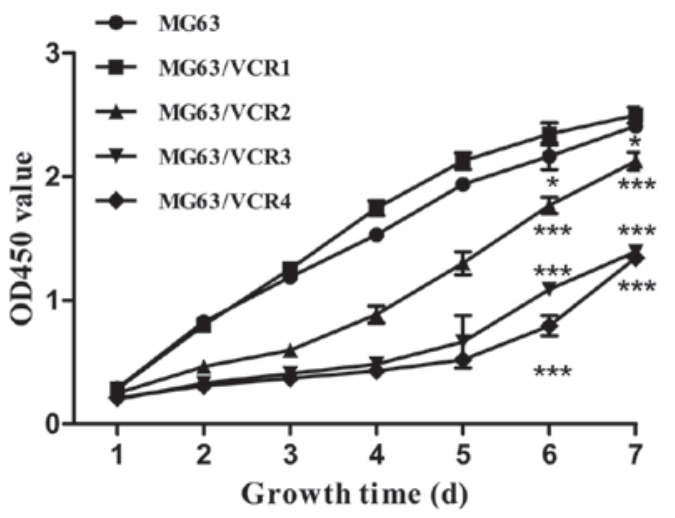

B

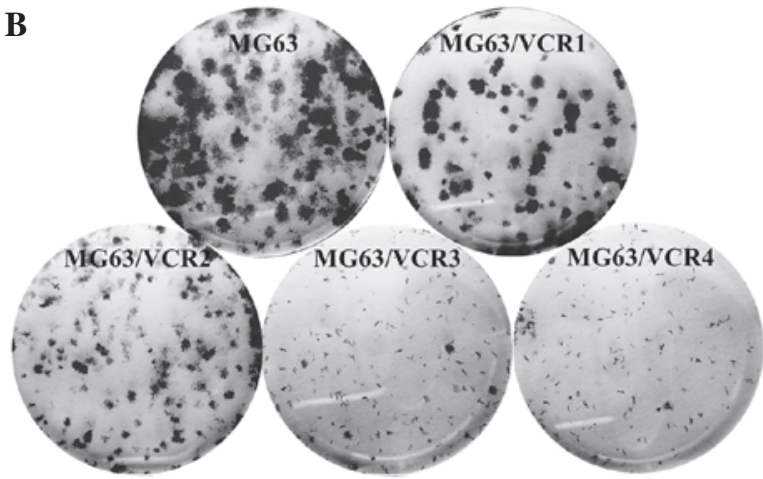

D
C

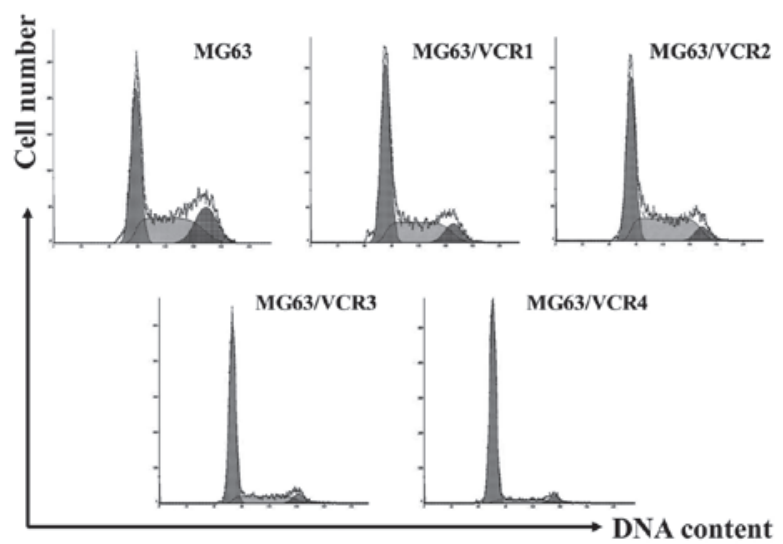

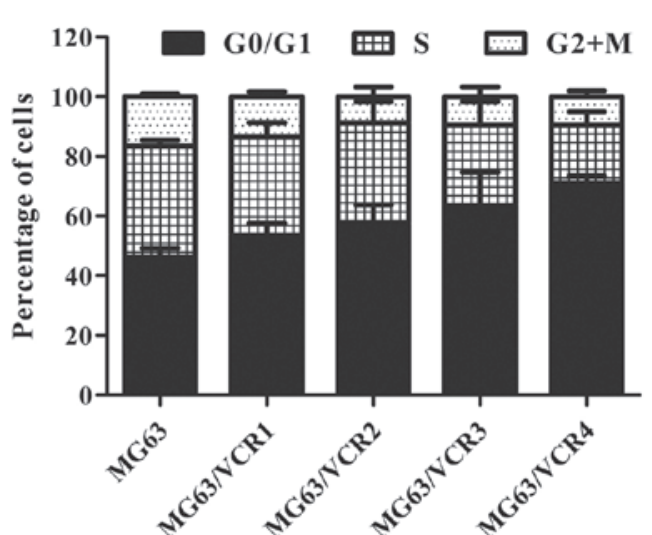

Figure 2. Differential proliferation capacity and cell cycle distribution of MG63 and MG63/VCR cells. (A) Cell Counting Kit-8 assay shows comparative analysis of cell growth between MG63 cells and sublines at the indicated time points. (B) Colonies formed 2 weeks after seeding are presented in the representative images. (C) Cell cycle analysis was performed by flow cytometry and (D) the data are presented in a histogram. Representative photographs are shown, data represent the mean \pm standard deviation of 5 independent experiments. ${ }^{*} \mathrm{P}<0.05,{ }^{* * *} \mathrm{P}<0.001$ vs. MG63. VCR, vincristine; OD, optical density.

oval in shape with a medium volume, and exhibited a distinct nucleolus with multiple nucleoli (Fig. 1A).

VCR-resistant osteosarcoma cell sublines exhibit resistance to different anticancer drugs. MG63/VCR cells exhibited less sensitivity to VCR-induced cell cytotoxicity compared with the parental cells. Specifically, the $\mathrm{IC}_{50}$ values of MG63 and MG63/VCR1, 2, 3 and 4 cell sublines to VCR were 0.952 and $155,453,1,182$ and $2,578 \mathrm{ng} / \mathrm{ml}$, respectively (Fig. 1B and C). The RIs of the derivative-resistant sublines $1,2,3$ and 4 were 163, 476, 1,242, and 2,708-fold higher than the parent MG63 cells, respectively. Notably, MG63/VCR cells also demonstrated a cross-resistance to DOX, PTX and THP, and a weak resistance to MTX, however the cells were sensitive to GEM (Fig. 1D).

VCR-resistant osteosarcoma cell sublines exhibit reduced proliferation capacity. MG63/VCR cell growth was reduced compared with the parental MG63 cells (Fig. 2A) and the colonies formed were smaller (Fig. 2B), although the colony formation ratio exhibited no significant difference (data not shown). Cell cycle distribution was assessed in the VCR-resistant osteosarcoma cell sublines using flow cytometry. As demonstrated in Fig. 2C and D, there were higher percentages of MG63/VCR cells in the G0/G1 phase and less in the S phase compared with parental MG63 cells, which is in accordance with the drug RI $(\mathrm{P}<0.05)$.
VCR-resistant osteosarcoma cell sublines exhibit differential migration capacity. A cell wound-healing assay was performed to compare the migration ability of MG63/VCR and MG63 cells. Remarkably, we found slower migration ability of MG63/VCR2, MG63/VCR3, and MG63/VCR 4 cells compared to parental MG63 cells (Fig. 3A and B). Notably, MG63/VCR1 showed a markedly increased migration ability when compared to MG63 cells or other sublines.

VCR-resistant osteosarcoma cell sublines exhibit differential expression of drug resistance-associated genes. RT-qPCR analysis of different drug resistance-associated genes was performed and indicated that MDR1, MRP1, topoisomerase II (TOPO-II), and LIM domain kinase 1 (LIMK1) mRNA in MG63/VCR cells were expressed at higher levels compared with MG63 cells $(\mathrm{P}<0.01)$. However, in the highly resistant MG63/VCR3 and MG63/VCR4 cells, the levels of MRP1, TOPO-II, and LIMK1 were significantly reduced compared with the moderately resistant MG63/VCR2 cells. Additionally, differential expression of GST- $\pi$ mRNA was not detected between MG63/VCR and MG63 cells (Fig. 4B).

MDR1 is involved in the resistance of the osteosarcoma cell sublines. MG63/VCR4 and MG63/VCR3 cells were highly resistant to VCR treatment compared with MG63 cells (Fig. 5A). MG63/VCR3 cells were selected for use in the verapamil rescue 


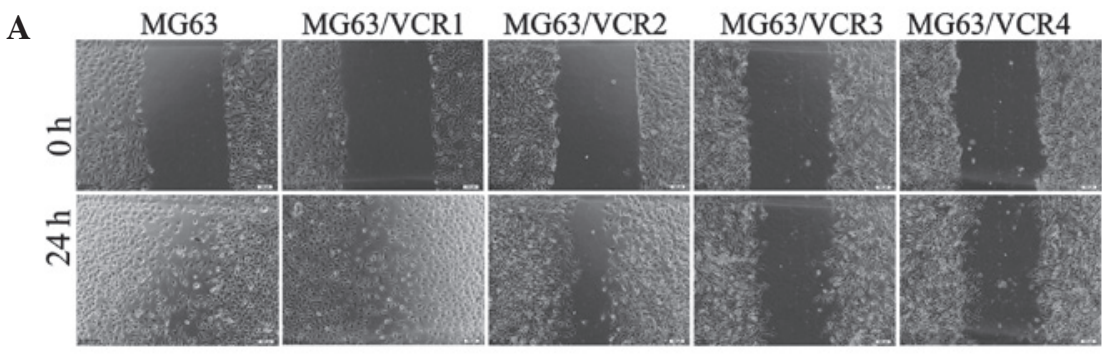

B

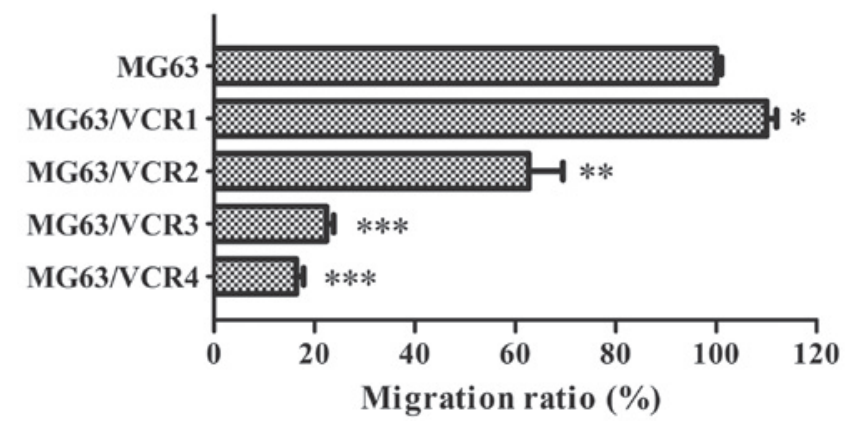

Figure 3. Differential migration capacity of MG63 and MG63/VCR cells. (A) In vitro wound healing assay of MG63 and MG63/VCR cells. The time-based images were obtained from 0 to $24 \mathrm{~h}$. (B) The relative migration distance of MG63/VCR sublines compared with the parental MG63 cells. Representative images are presented and data represent the mean \pm standard deviation of 6 independent experiments. ${ }^{*} \mathrm{P}<0.05,{ }^{* * *} \mathrm{P}<0.01$ and ${ }^{* * * *} \mathrm{P}<0.001$ vs. MG63. VCR, vincristine.

A

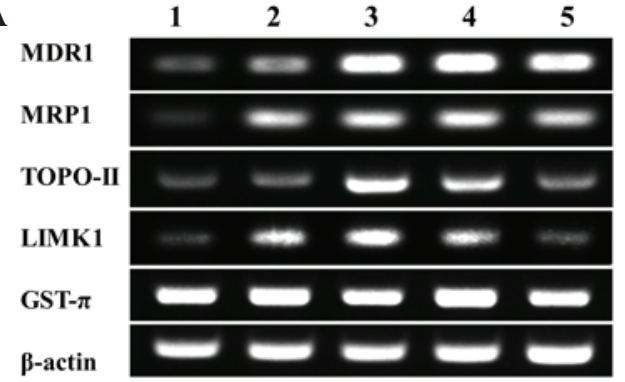

B

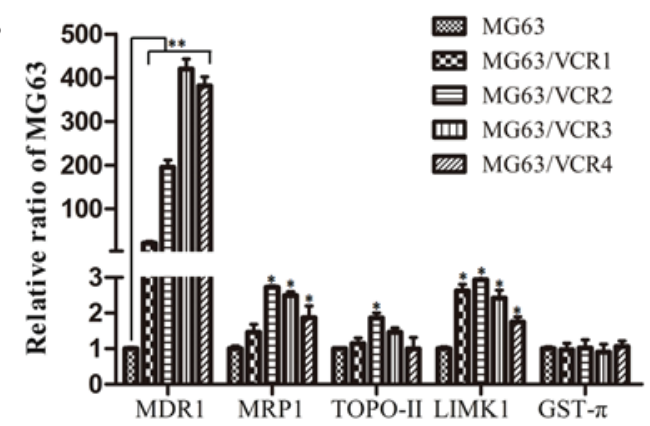

Figure 4. Gene expression of MG63 and MG63/VCR cells. (A) RT-PCR. Expression of drug resistance-associated genes was analyzed by RT-PCR and agarose gel electrophoresis of PCR products of MDR1, MRP1, TOPO-II, LIMK1, GST- $\pi$, and $\beta$-actin. Lanes 1-5 represent MG63, MG63/VCR1, MG63/VCR2, MG63/VCR3, and MG63/VCR4, respectively. (B) Expression levels of each mRNA were quantitatively analyzed and normalized relative to $\beta$-actin level. Data represent the mean \pm standard deviation of 5 independent experiments. ${ }^{*} \mathrm{P}<0.05,{ }^{* *} \mathrm{P}<0.01$ vs. the MG63 group. RT-PCR, reverse transcription polymerase chain reaction; MDR1, multidrug resistance protein 1; MRP1, MDR-associated protein 1; TOPO-II, topoisomerase II; LIMK1, LIM domain kinase 1; GST- $\pi$, glutathione S-transferase- $\pi$; VCR, vincristine.

assay. Subsequent to the addition of verapamil (a calcium channel blocker, which blocks the function of MDR1), highly resistant MG63/VCR3 cells became sensitive to VCR and this change was dependent on the verapamil concentrations (Fig. 5B). DOX is auto-fluorescent with the same wave-length as PI (20). Thus, the efflux DOX was measured in the VCR-resistant sublines. The data indicated that DOX accumulation was higher in MG63 cells compared with MG63/VCR1-4 sublines as demonstrated by decreased DOX fluorescence intensity in MG63/VCR cells (Fig. 5C and D). However, following treatment with verapamil, DOX fluorescence intensity increased in the MG63/VCR cells compared with DOX-only treatment.

\section{Discussion}

Chemotherapy combined with surgery is the principal method of treatment for human cancer. However, tumor resistance to chemotherapy and molecular targeted therapies limit the effectiveness of current drug efficacy $(1,21)$. Cancer cells can become cross-resistant to a broad spectrum of chemotherapeutic agents with different biological characteristics and structure following a duration of single drug treatment, termed MDR (8). At the cellular level, various MDR mechanisms are involved in chemoresistance, including increase in drug efflux, mutations of the drug targeting genes, DNA repair capacity, activation of alternative signaling pathways or evasion of cell death (21). It is increasingly recognized that tumor lesions contain a high degree of molecular heterogeneity (13) and that drug resistant tumor cells may enhance a therapy-induced selection of a resistant minor subpopulation of cells for expansion $(21,22)$. However, the precise mechanisms of MDR remain undefined and require further investigation (21). The current study established a series of VCR-resistant osteosarcoma cell lines (MG63/VCR1, MG63/VCR2, MG63/VCR3, and 
A

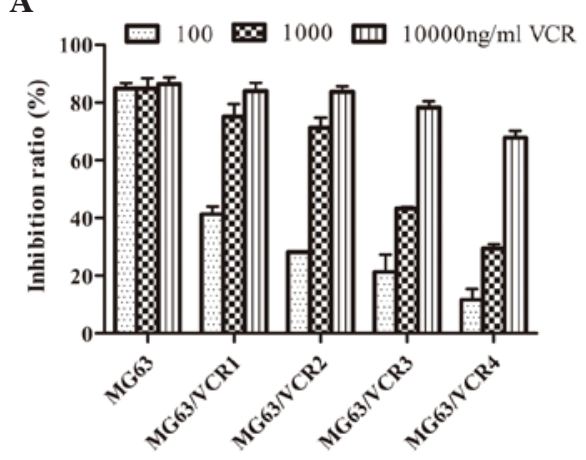

B

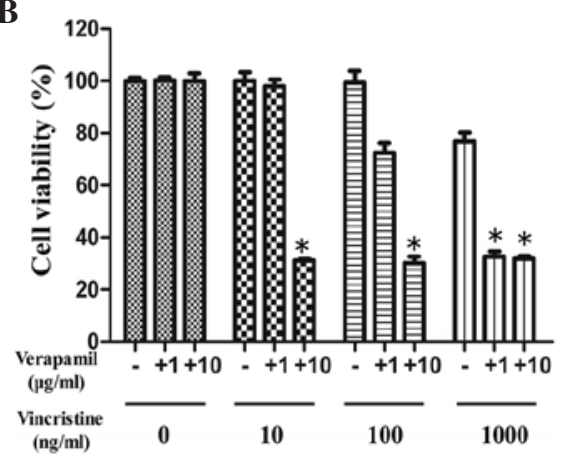

$\mathbf{C}$
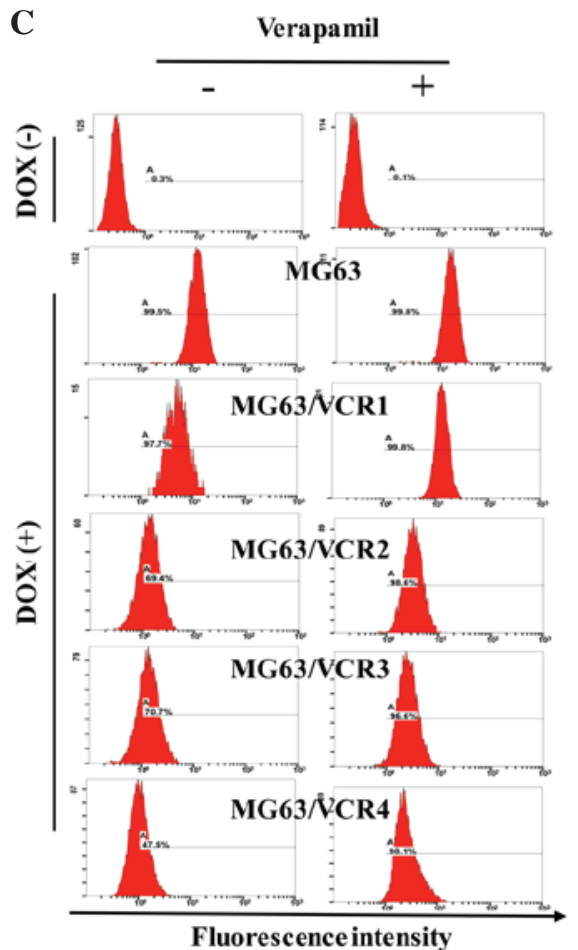

D

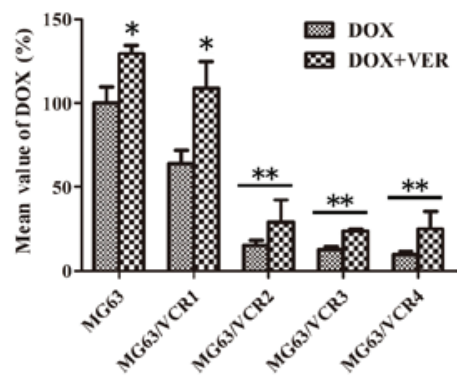

E
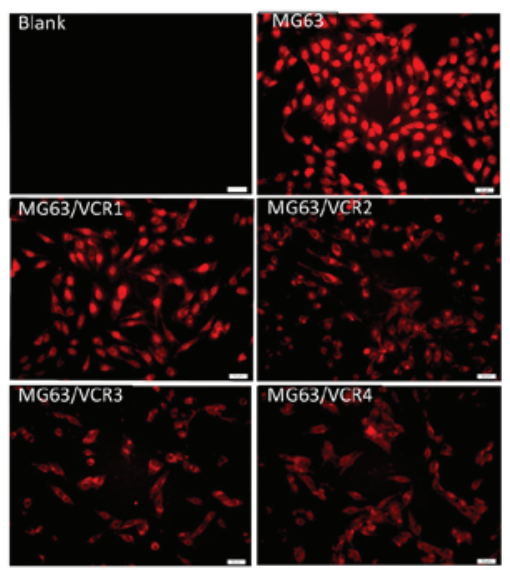

Figure 5. Multidrug resistance protein 1 is important in multidrug resistance. (A) CCK-8 assay demonstrated that VCR suppressed cell proliferation. Cells were incubated for $48 \mathrm{~h}$ in the presence of different concentrations of VCR $(0.1,1$ or $10 \mu \mathrm{g} / \mathrm{ml})$. (B) The CCK-8 assay demonstrated that VER increased tumor cell resistance to VCR in MG63/VCR3. Data represent the mean \pm standard deviation of 5 independent experiments. (C) Fluorescence intensity of DOX was quantitatively analyzed by fluorescence-activated cell sorting and (D) the mean intensity of the fluorescence is presented in the bar graph. (E) Intracellular accumulation of DOX was also evaluated by fluorescence microscopy. The experiments were repeated 3 times with similar results. ${ }^{*} \mathrm{P}<0.05$, ${ }^{* *} \mathrm{P}<0.01$ vs. the control group. Scale bar=50 $\mu \mathrm{m}$. CCK-8, cell counting kit-8; VCR, vincristine; DOX, doxorubicin; VER, verapamil.

MG63/VCR4) with typical MDR phenotypes. Compared with parental MG63 cells, MG63/VCR cells exhibited different RI to VCR and cross-resistance to other structurally and mechanistically different drugs (Fig. 1), including an antimicrotubule agent (PTX), antimetabolite agent (MTX) and TOPO-II inhibitors (DOX and THP). All these drugs are frequently used in the clinic as chemotherapeutics for osteosarcoma $(1,3)$. The MDR characteristics of MG63/VCR cells may indicate the failure of the chemotherapy combination of VCR with DOX, PTX or THP in clinical practice $(3,23)$. However, the data of the present study also demonstrated that these cell sublines were sensitive to GEM, which is a nucleoside analog, and functions to arrest tumor growth and induce tumor cell apoptosis. Sensitivity of these sublines to GEM may be attributed to collateral sensitivity (24).

Morphologically, the VCR-resistant cells are distinct from their parental cell line. For example, the resistant cells exhibited spindle-shaped morphology and increased formation of pseudopodia. The long cytoplasmic processes at the opposite poles of the cell were increased with increased RI (Fig. 1A), which is consistent with the findings of a previous study in different tumor cells (25). Wen et al (26) reported that ultrastructural changes may facilitate survival of drug-resistant cells during and after chemotherapy. Furthermore, drug-resistant tumor cells may exhibit different abilities in cell proliferation and migration, and additionally other studies demonstrated different and even contradictory results (9-12). In the current study, the proliferation ability of MG63/VCR cells was significantly decreased
(Fig. 2) with increased RI. Park et al (27) suggested that reduced proliferation may be caused by an increased number of quiescent cancer cells or non-cycling dormant cells, characterized by resistance to therapy (27). This conjecture has been supported by cell cycle analysis, with the percentages of MG63/VCR cells in the $G_{0} / G_{1}$ phases significantly increased compared with the percentages in the MG63 cells in the corresponding phases (Fig. 2C and D). MDR and metastasis are two signs of malignant tumor. The migration ability was significantly enhanced in the lower resistance MG63/VCR1 cells compared with the MG63 cells. However, migration was significantly reduced in the highly resistant MG63/VCR3 and MG63/VCR4 cells (Fig. 3A and B). The expression of certain genes is consistent with this reduction in migration, such as LIMK1, which is important for the regulation of the actin cytoskeleton, thus promoting tumor cell migration and invasion. Different sublines with different RI demonstrated divergent drug-resistant mechanisms. Therapeutic intervention provides a potent selective pressure for the survival of tumor cells, and a subpopulation will survive and become resistant to the drugs, which will inherit their biological characteristics and replace the original tumor clones; this is analogous to Darwinian evolution theory $(13,28)$.

Although the precise mechanism of MDR remains unclear, several cell membrane transporter proteins have associated resistance with commonly used chemotherapeutics that promote drug efflux. Notably, the ATP-binding cassette transporter family of transmembrane proteins regulate the efflux of multiple structurally and mechanistically unrelated chemotherapeutic 
agents across the plasma membrane (21). Several members of this protein family have been extensively studied, including MDR1 and MRP1. Furthermore, GST enables detoxification of endogenous compounds and the metabolism of xenobiotics (29). TOPO-II and LIMK1 are also understood to be associated with drug resistance and tumor cell motility (30-32). In this context, drug-resistant cells overexpress these genes. However, certain studies have demonstrated downregulation of several MDR-associated genes, including MDR1 and MRP1 in drug-resistant cancer cell lines $(33,34)$, indicating the involvement of a different drug resistance mechanism. The data of the current study demonstrated that with increased RI, MDR1 mRNA expression was raised and plateaued, whereas MRP1, LIMK1 and TOPO-II levels were highly expressed in the moderately resistant MG63/VCR2 cells, whereas their expression was lower in the highly resistant MG63/VCR3 and MG63/VCR4 cells (Fig. 4A and B). These data suggested that the mechanism of drug resistance in cells lines with different drug resistance levels may be different. Additionally, the current study assessed the retention level of DOX in the cells, and then challenged these cells with the MDR1 antagonist, verapamil (2-6 $\mu \mathrm{M}$ of which is sufficient to block MDR1 activity). DOX was used because it exhibits auto-fluorescence at the same wavelength as PI (20). The data demonstrated that the level of DOX was reduced in these sublines with increased RI (Fig. 5). Following verapamil treatment, the level of DOX in the cells was markedly increased, suggesting that MDR1-associated drug resistance was associated with a marked reduction in DOX accumulation and may be an important mechanism by which MG63/VCR sublines are resistant to chemotherapeutics. Further studies are required to assess how MDR1 activity affects MDR cell sublines.

In conclusion, the current study established stable MG63/VCR MDR osteosarcoma cell sublines, which were cross-resistant to other drugs. These sublines may serve as a useful tool for further study of the molecular mechanisms of osteosarcoma drug resistance and novel therapeutic strategies for osteosarcoma.

\section{Acknowledgements}

This study was supported by Projects of International Cooperation of Jilin Provincial Science \& Technology Department (grant no. 20150101175JC) and the National Natural Science Foundation of China (grant nos. 81172000 and 30772488).

\section{References}

1. Luetke A, Meyers PA, Lewis I and Juergens H: Osteosarcoma treatment-where do we stand? A state of the art review. Cancer Treat Rev 40: 523-532, 2014.

2. Ottaviani $\mathrm{G}$ and Jaffe N: The epidemiology of osteosarcoma. Cancer Treat Res 152: 3-13, 2009.

3. Ta HT, Dass CR, Choong PF and Dunstan DE: Osteosarcoma treatment: State of the art. Cancer Metastasis Rev 28: 247-263, 2009.

4. Ferrari S and Palmerini E: Adjuvant and neoadjuvant combination chemotherapy for osteogenic sarcoma. Curr Opin Oncol 19: 341-346, 2007

5. Panda S, Choudhary K, Srivastava G, Padhiary SK, Dhull KS and Sanghavi D: An Indian perspective on gnathic osteosarcoma: A comprehensive literature review of the last three decades J Oral Maxillofac Surg Med Pathol 26: 198-206, 2014.

6. Picci P: Osteosarcoma (Osteogenic sarcoma). Orphanet J Rare Dis 2: 6, 2007.
7. Rosen G: Neoadjuvant chemotherapy for osteogenic sarcoma: A model for the treatment of other highly malignant neoplasms. Recent Results Cancer Res 103: 148-157, 1986.

8. Thomas $\mathrm{H}$ and Coley HM: Overcoming multidrug resistance in cancer: An update on the clinical strategy of inhibiting p-glycoprotein. Cancer Control 10: 159-165, 2003.

9. Niu BH, Wang JJ, Xi Y and Ji XY: The establishment and characterization of adriamycin-resistant cell lines derived from Saos-2. Med Sci Monit 16: BR184-BR192, 2010.

10. Okugawa K, Kobayashi H, Hirakawa T, Sonoda T, Ogura T and Nakano H: In vivo establishment and characterization of a paclitaxel-resistant human ovarian cancer cell line showing enhanced growth properties and drug-resistance only in vivo. J Cancer Res Clin Oncol 130: 178-186, 2004.

11. Kanzawa F, Sugimoto Y, Minato K, Kasahara K, Bungo M, Nakagawa K, Fujiwara Y, Liu LF and Saijo N: Establishment of a camptothecin analogue (CPT-11)-resistant cell line of human non-small cell lung cancer: Characterization and mechanism of resistance. Cancer Res 50: 5919-5924, 1990.

12. Negoro K, Yamano Y, Fushimi K, Saito K, Nakatani K, Shiiba M, Yokoe H, Bukawa H, Uzawa K, Wada T, et al: Establishment and characterization of a cisplatin-resistant cell line, KB-R, derived from oral carcinoma cell line, KB. Int J Oncol 30: 1325-1332, 2007.

13. Swanton C: Intratumor heterogeneity: Evolution through space and time. Cancer Res 72: 4875-4882, 2012.

14. Almendro V, Marusyk A and Polyak K: Cellular heterogeneity and molecular evolution in cancer. Annu Rev Pathol 8: 277-302, 2013.

15. McGranahan $\mathrm{N}$ and Swanton C: Biological and therapeutic impact of intratumor heterogeneity in cancer evolution. Cancer Cell 27: 15-26, 2015.

16. Burrell RA, McGranahan N, Bartek J and Swanton C: The causes and consequences of genetic heterogeneity in cancer evolution. Nature 501: 338-345, 2013.

17. Barteneva NS, Ketman K, Fasler-Kan E, Potashnikova D and Vorobjev IA: Cell sorting in cancer research-diminishing degree of cell heterogeneity. Biochim Biophys Acta 1836: 105-122, 2013.

18. Zhang H, Wang Y, Xing F, Wang J, Wang Y, Wang H, Yang Y and Gao Z: Overexpression of LIMK1 promotes migration ability of multidrug-resistant osteosarcoma cells. Oncol Res 19: 501-509, 2011.

19. Livak KJ and Schmittgen TD: Analysis of relative gene expression data using real-time quantitative PCR and the 2(-Delta Delta C(T)) method. Methods 25: 402-408, 2001.

20. Zhang X, Peng X, Yu W, Hou S, Zhao Y, Zhang Z, Huang X and $\mathrm{Wu} \mathrm{K}$ : Alpha-tocopheryl succinate enhances doxorubicin-induced apoptosis in human gastric cancer cells via promotion of doxorubicin influx and suppression of doxorubicin efflux. Cancer Lett 307: 174-181, 2011

21. Holohan C, Van Schaeybroeck S, Longley DB and Johnston PG: Cancer drug resistance: An evolving paradigm. Nat Rev Cancer 13: 714-726, 2013.

22. Greaves M and Maley CC: Clonal evolution in cancer. Nature 481: 306-313, 2012.

23. Souhami RL, Craft AW, Van der Eijken JW, Nooij M, Spooner D, Bramwell VH, Wierzbicki R, Malcolm AJ, Kirkpatrick A, Uscinska BM, et al: Randomised trial of two regimens of chemotherapy in operable osteosarcoma: A study of the European Osteosarcoma Intergroup. Lancet 350: 911-917, 1997.

24. Cerezo D, Cánovas M, García-Peñarrubia P and Martín-Orozco E: Collateral sensitivity to cold stress and differential BCL-2 family expression in new daunomycin-resistant lymphoblastoid cell lines. Exp Cell Res 331: 11-20, 2015.

25. Takeshita H, Kusuzaki K, Ashihara T, Gebhardt MC, Mankin HJ and Hirasawa Y: Intrinsic resistance to chemotherapeutic agents in murine osteosarcoma cells. J Bone Joint Surg Am 82-A: 963-969, 2000

26. Wen J, Zheng B, Hu Y, Zhang X, Yang H, Luo KJ, Zhang X, $\mathrm{Li} \mathrm{YF}$ and Fu JH: Establishment and biological analysis of the EC109/CDDP multidrug-resistant esophageal squamous cell carcinoma cell line. Oncol Rep 22: 65-71, 2009.

27. Park J, Bae EK, Lee C, Choi JH, Jung WJ, Ahn KS and Yoon SS Establishment and characterization of bortezomib-resistant U266 cell line: Constitutive activation of NF-kB-mediated cell signals and/or alterations of ubiquitylation-related genes reduce bortezomib-induced apoptosis. BMB Rep 47: 274-279, 2014.

28. Gerlinger M and Swanton C: How Darwinian models inform therapeutic failure initiated by clonal heterogeneity in cancer medicine. Brit J Cancer 103: 1139-1143, 2010. 
29. Kars MD, Iseri ÖD and Gündüz U: A microarray based expression profiling of paclitaxel and vincristine resistant MCF-7 cells. Eur J Pharmacol 657: 4-9, 2011.

30. Chen Q, Jiao D, Hu H, Song J, Yan J, Wu L and Xu LQ: Downregulation of LIMK1 level inhibits migration of lung cancer cells and enhances sensitivity to chemotherapy drugs. Oncol Res 20: 491-498, 2013.

31. Zhou Y, Su J, Shi L, Liao Q and Su Q: DADS downregulates the Rac1-ROCK1/PAK1-LIMK1-ADF/cofilin signaling pathway, inhibiting cell migration and invasion. Oncol Rep 29: 605-612, 2013.
32. Zhang HS, Zhao JW, Wang H, Zhang HY, Ji QY, Meng LJ, Xing FJ, Yang ST and Wang Y: LIM kinase 1 is required for insulin-dependent cell growth of osteosarcoma cell lines. Mol Med Rep 9: 103-108, 2014.

33. Yan XD, Li M, Yuan Y, Mao N and Pan LY: Biological comparison of ovarian cancer resistant cell lines to cisplatin and Taxol by two different administrations. Oncol Rep 17: 1163-1169, 2007.

34. Li L, Luan Y, Wang G, Tang B, Li D, Zhang W, Li X, Zhao J, Ding H, Reed E and Li QQ: Development and characterization of five cell models for chemoresistance studies of human ovarian carcinoma. Int J Mol Med 14: 257-264, 2004. 